\title{
Desenvolvimento de uma ferramenta de avaliação para aplicação do Mindful Design no processo de desenvolvimento de produtos
}

\author{
Designing an evaluation tool to apply Mindful Design into design process
}

BOSSE, Michaelle; Estudante de Doutorado; Technische Universität Dresden (TU Dresden) michaellebosse@gmail.com

\section{Resumo}

Este artigo relata o desenvolvimento de uma ferramenta de avaliação de Mindful Design para uso no contexto do ensino superior em design industrial. É um estudo de caso que avalia conceitos de produtos desenvolvidos por estudantes de design para pessoas com demência precoce no contexto de uso pessoal. A estrutura e o conteúdo da ferramenta de avaliação desenvolvida foram baseados em uma revisão de literatura sobre Mindfulness, teoria em que o Mindful Design se baseia. Aspectos como consciência, atenção, novidade, engajamento e flexibilidade, construtos importantes para o estado de consciência sócio-cognitiva, são os critérios básicos para a formulação dessa ferramenta. Destes, foram selecionadas afirmações de avaliações de auto-questionamento já existentes de Mindfulness para classificar e medir o nível de atenção plena gerado durante a interação com produtos conscientes. Finalmente, esta ferramenta de avaliação foi testada por um grupo de especialistas (designers e técnicos de saúde), a fim de rever quais declarações seriam apropriadas para o design e a interação dos produtos.

Palavras Chave: Mindful Design, Mindfulness, ferramenta de avaliação, ensino superior em design.

\begin{abstract}
This paper reports the development of a mindful design evaluation tool for use in the context of industrial design higher education. It is a study case which evaluate products concepts developed by design students for people with initial and middle level of dementia in the context of personal use. The structure and content of the developed evaluation tool was based on a literature review of Mindfulness, the ground-based concept of Mindful Design. Aspects such as Awareness, Attention, Novelty, Engagement and Flexibility, important constructs for the socio-cognitive mindfulness state, are the ground criteria of the formulation of this tool. From these, we have selected statements from existing Mindfulness self-report assessments to classify and measure the level of mindfulness generated during the interaction with mindful products. Finally, this evaluation tool was tested by a group of experts (designers and heath care), in order to review which statements would be suitable for the design and interaction of products.
\end{abstract}

Keywords: Mindful Design, Mindfulness, evaluation tool, design higher education. 


\section{Introdução}

A ampla revisão da literatura sobre mindfulness considera, majoritariamente, seus potenciais benefícios na saúde física e mental, mas não se podem esquecer os fatores para o bemestar psicológico. Estudos sobre empatia (SHAPIRO et al., 1998; AU, 2015), busca de novidades, produção de novidades, engajamento e flexibilidade (BODNER; LANGER, 2001), aumento do desempenho cognitivo e compaixão (LIM et al., 2015) e melhoria da cognição social (VAGO; SILBERSWEIG, 2012), são algumas das interpretações ocidentais dos benefícios dos mecanismos de mindfulness.

A literatura reconhece duas correntes predominantes de pesquisa e prática de mindfulness: mindfulness meditativo para tratamento e redução do estresse (KABAT-ZINN, 2003) e mindfulness cognitivo para melhoria do desempenho cognitivo (LANGER, 1990). Um lado define mindfulness como "a consciência que emerge ao propositalmente prestar atenção no momento presente e sem julgar o desdobramento da experiência a cada momento" (KABAT-ZINN, 2003, p. 145). Essa abordagem está relacionada às práticas meditativas Budistas e considera que o mindfulness afeta positivamente a atenção, que aumentaria ao longo do processo de manter a prática diária de meditação (GUNARATANA, HENEPOLA, 2012; KABAT-ZINN, 2003). O outro lado da literatura defende, assim como a primeira abordagem, o compromisso com a noção de estar no momento presente, relatado pelos estudos de Langer sobre falta de atenção e escolha. Mas, essa abordagem apresenta mindfulness como o processo de ativamente fazer novas distinções por não depender de categorizações automáticas (IE et al., 2014). O autor explica, a atenção plena é atualizada mantendo-se uma orientação no presente, alerta às distinções, uma sensibilidade aos diferentes contextos e uma abertura à novidade, bem como à criatividade (LANGER, 1990).

Imagem 1: Mapa mental das diversas áreas sobre a pesquisa Mindfulness, divididas nas baseadas em meditação e nas baseadas em teorias sócio-cognitivas

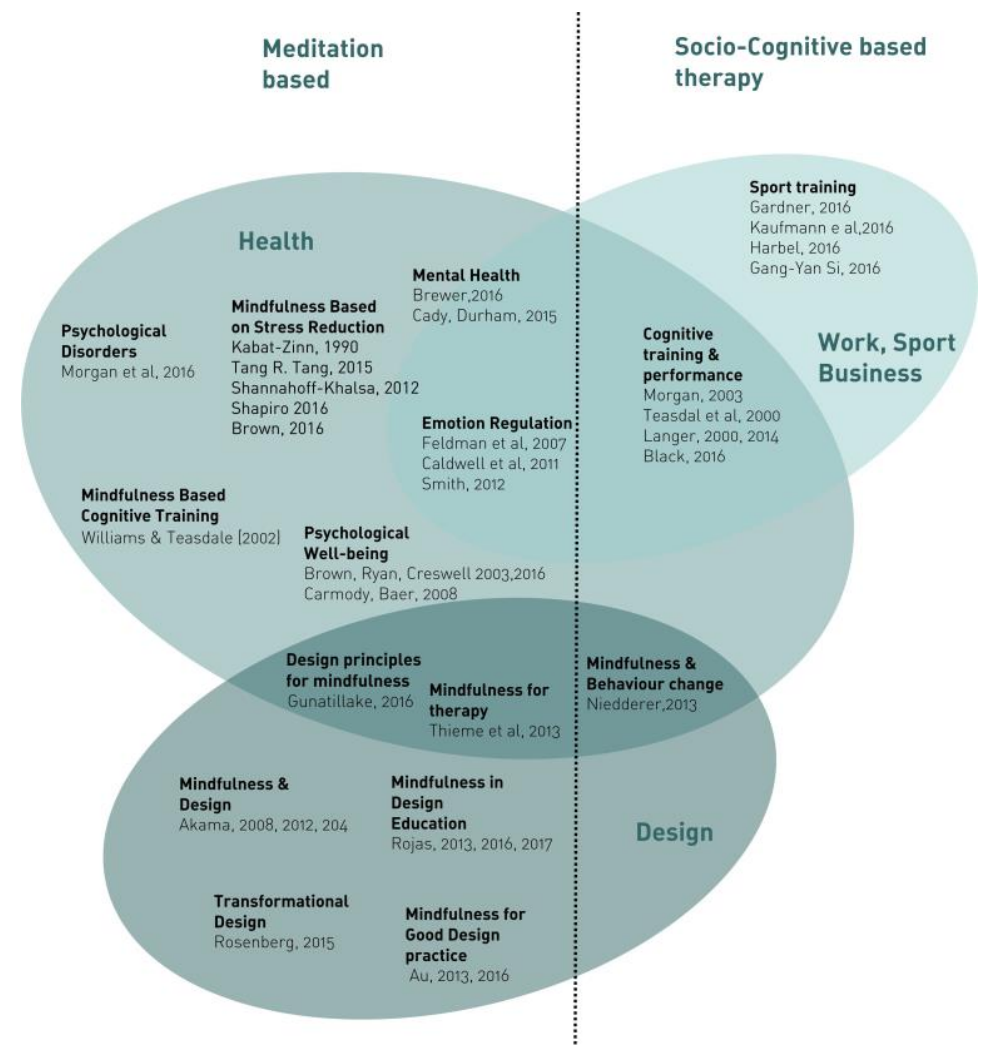


Os estudos de Djikic (2014) tiveram como objetivo ligar as abordagens de mindfulness entre o leste e o oeste. Para os estudos ocidentais, mindfulness visa abordar a ausência de consciência (LANGER, 1990, 2014) com associação da falta de escolhas por causa de categorias de produção em alta ou incompatibilidade entre categorias cognitivas profundamente enraizadas e questões do mundo emergente. Isso significa trabalhar e criar sob a égide de regras pré-concebidas que contrastam com a natureza mutável da realidade da vida, em uma abordagem não-meditativa. Langer (2000) explica que, em contraste com a atenção plena (mindfullness), a ausência de atenção (mindlessness) é descrita como uma ação automática que pode ser útil, pois libera a mente para executar níveis mais elevados de funcionamento cognitivo. O argumento contra a ausência de atenção é que a maioria do processamento de informações mentais parece não ter sentido, e estudos revelam que agir em "piloto automático" por tempo demais pode ser prejudicial para essas mesmas funções cognitivas. Enquanto que, para os estudos orientais, a raiz das práticas de mindfulness é contra o sofrimento que inclui não apenas a inevitabilidade da doença, velhice e morte, mas também a dor de frequentemente não conseguir o que queremos, e então sofrer sobre isso. A lição trata de como superar e resolver esses problemas, como obter resultados positivos disso, remediar tais questões começa e termina internamente, com técnicas de meditação do Oriente que apontam o caminho para o autodesenvolvimento através da quietude e do silêncio (WALSH; SHAPIRO, 2006). Finalmente, a prática da atenção plena é indicada para aumentar a concentração, a consciência no presente e construir uma condição de vida consciente.

\section{Mindfulness e ensino}

O conceito de atenção plena tem sido usado em avaliações de ensino na última década. Exemplificado por Yeganeh e Kolb (2009), relacionados aos estudos da teoria de aprendizagem experiencial, mindfulness é um estado em que o indivíduo:

1. centra-se na experiência presente e direta;

2. é intencionalmente consciente e atento;

3. aceita a vida como um processo emergente de mudança.

Vários autores denotam que a prática de mindfulness refere-se a mudar o modo de perceber a vida diária e as atividades familiares, com novos olhos e atenção (YEGANEH; KOLB, 2009; ROJAS et al., 2017, 2015, 2016; LANGER, 1990, 2000). Essa consciência de relacionalidade é relevante para um modelo útil de conscientização de um campo da Neurobiologia (VAGO; SILBERSWEIG, 2012). Os autores propuseram um modelo para operacionalizar o construto da atenção plena, bem como os seus mecanismos subjacentes. Em seu estudo, eles forneceram uma estrutura teórica integrativa e um modelo neurobiológico baseado em sistemas que explicam os mecanismos pelos quais a atenção reduz os vieses relacionados ao autoprocessamento e cria uma sustentável mente saudável. Isso implica que, embora todos os efeitos sugeridos da atenção plena tenham relevância relacional, é através da transformação da consciência interior que isso é realizado (ROJAS et al., 2015)

Ao mudar a mentalidade, mudar a perspectiva e experimentar a consciência no presente, a criatividade, a flexibilidade e, consequentemente, o bem-estar psicológico e a saúde física podem aumentar. 


\section{Mindful Design}

Com a introdução de métodos como o Design Thinking, Howard e Melles (2011) e a cocriação/participação de Sander e Stappers (2008) no processo de design, os usuários tiveram um papel ativo e importante na resolução de problemas do projeto. Assim como esses métodos, o mindfulness tem um papel importante em ambos os processos: levar a estimulação cognitiva ao processo de aprendizado do design e ao próprio processo de design. Roja (2015) e Rosenberg (2015) argumentam que a integração da atenção plena no contexto do design pode ajudar não apenas os designers a manterem-se conscientes das necessidades do usuário, mas também criar um estado de consciência interna que permita um relacionamento consciente com os outros e com o ambiente. Além disso, Niedderer (2013) comenta que a introdução das características sócio-cognitivas da atenção plena no processo de design pode fazer com que o produto promova essa mudança de mentalidade na vida diária, levando consequentemente à mudança de comportamento no ambiente.

Isso muda a maneira de projetar. Em vez de apenas tentar entender as necessidades do usuário, como sempre foi feito nos processos de design clássicos, ele também os torna parte da tomada de decisões do projeto. Designers se tornam responsáveis por sua contribuição e conscientes de suas escolhas.

Por essas razões, a introdução de mecanismos de mindfulness para o ensino pode esclarecer tanto o processo de aprendizagem quanto o de design. Isso pode ajudar os alunos a entender como - Mindful Design pode estimular a consciência (para si / para o outro / para o ambiente), o foco, a criatividade, o engajamento e a flexibilidade nas atividade de vida cotidianas.

O conceito de mindfulness no design, também denominado design consciente por Niedderer (2004, 2014), refere-se à atenção do usuário em relação às consequências de suas ações realizadas com um objeto (IE et al., 2014). O autor afirma nesses estudos que um artefato pode ser projetado de tal forma que estimule a atenção plena quando um contexto consciente não está disponível (Niedderer, 2004, 2007, 2013).

Ligando o design consciente com a atenção plena como prática, é possível ver semelhanças em sua definição tradicional de meditação, que é uma maneira de prestar atenção e ver claramente o que está acontecendo em nossas vidas. A atenção prestada é intencional, no momento, sem julgamento. Trata-se de ter tempo e espaço para prestar atenção ao que nos rodeia, apesar das distrações que competem por nossa atenção e nossa história que molda a forma como pensamos e percebemos o mundo (AU, 2015).

Para Au (2015) o atributo de um design consciente, muito semelhante à prática de meditação e ações meditativas como ioga, é oferecer aos usuários a possibilidade de experimentar paz, equilíbrio, consciência, foco, empatia, desapego.

\section{Mindful design: Interação Social, Engajamento e Mudança Comportamental}

Considerando mindfulness como uma forma transformadora de projetar, de manter a consciência dos designers no propósito do artefato para os usuários, podemos encontrar exemplos em muitos contextos, como o ambiente construído e produtos de consumo digital, incluindo telefones celulares, móveis e utensílios de mesa. $O$ uso de artefatos também pode afetar a interação social de maneiras desejáveis ou indesejáveis e/ou para que ela seja atenta ou desatenta (LANGER, 1989; IE et al., 2014). 
Niedderer (2014) exemplifica o objeto performativo para mindfulness, que tem o efeito de interromper a consciência do usuário para a ação automática e requer um alerta adicional para o usuário completar a ação, como design de jogos, dispositivos de segurança ou avisos em computadores (por exemplo, ao salvar um documento). Ainda, outro exemplo identificado como objeto performativo é a bancada "Come a little closer", projetada em 2001 por Nina Farkache da Droog Design. Ela permite que os usuários interajam fisicamente entre si, quebrando a expectativa de apenas sentar no banco para descansar, mas também para experimentar a interação com outros usuários por curiosidade e diversão (IE et al., 2014).

\section{Avaliações de Mindfulness por autorrelato}

A atenção plena é um campo amplo, que vem aumentando o interesse em avaliações adequadas para melhorar o tratamento psicológico. Além disso, a avaliação da atenção plena, tem levado à vários métodos para operacionalizar e medir o uso clínico, baseado principalmente em uma perspectiva budista oriental (PIRSON et al., 2012).

Alguns dos critérios de avaliação de mindfulness por autorrelato são a compreensão psicológica de estado e característica pessoal. As diferenças entre as duas características são consideradas na discussão abaixo.

\subsection{Diferenciação da compreensão psicológica de estado e característica pessoal}

Com o aumento da aplicação de intervenções baseadas em mindfulness, a avaliação exata de ambos, a tendência geral para ser atento (um traço) e o grau de atenção do indivíduo em qualquer momento específico (um estado), tornou-se uma questão importante de estudo clínico e de pesquisa (MEDVEDEV et al., 2017, p. 1036).

Pesquisadores no campo da psicologia discutiram as diferenças entre as características do estado e do traço ao longo dos anos. Basicamente, o estado é criado por uma mudança emocional temporária. É definido como uma reação emocional a fatores internos e/ou externos que influenciam alterações físicas, comportamentais, cognitivas e psicológicas. Dependendo do nível de excitação, frustração, percepção subjetiva e contexto, pode estipular a duração e a intensidade da emoção sentida (GALOR, 2012).

O traço, por outro lado, é uma faceta mais permanente da personalidade, implicando uma duração mais perene e um nível estável de emoção. Os traços descrevem as características de personalidade do indivíduo e que são constantes ao longo do tempo, por exemplo, se a pessoa é muito ansiosa, tímida ou irritada. Basicamente, traços interagem com diferentes fatores para criar muitos estados emocionais, incluindo extroversão, autoestima, perfeccionismo e impulsividade. Embora existam algumas formas de terapia ou modificação de comportamento que possam afetar essas características, elas geralmente não mudam sem um esforço conjunto (SPIELBERGER; SYDEMAN, 1994).

Para Ackerman (2017), o estado de mindfulness refere-se a uma condição temporária de consciência que um indivíduo sente, observando seus pensamentos, sentimentos e sua capacidade de permanecer no momento presente, mesmo quando surgem distrações. E traço de mindfulness é a capacidade de atingir esse estado com mais frequência, mantendo o foco no presente por um longo período de tempo.

\subsection{Diferenças na Categorização de Mindfulness}


Os respectivos métodos de autorrelato são desenvolvidos para avaliar uma tendência geral à atenção plena na vida diária e também apresentam características psicométricas promissoras. Embora diferenças em seu conteúdo e estrutura sugiram uma falta de consenso entre os pesquisadores sobre se a atenção plena deve ser conceituada como um construto multifacetado e, em caso afirmativo, como as facetas devem ser definidas e operacionalizadas (BAER et al, 2008).

Tabela 1 - Dimensões de Mindfulness.

\begin{tabular}{|c|c|c|}
\hline \multicolumn{3}{|c|}{ Mindfulness dimensions } \\
\hline Acceptance & $\begin{array}{l}\text { Some authors mean acceptance as non-judgment. Some author split } \\
\text { these mechanisms in two different constructs. }\end{array}$ & $\begin{array}{l}\text { Bergomi, C; Tschacher, W. } \\
\text { \&Kupper, Z. (2014); } \\
\text { Cardatiotto, L. et al (2008); } \\
\text { Feldman, G. et al (2007); Baer, } \\
\text { R. A., Smith G. T., \& Allen, KB. } \\
\text { (2004) }\end{array}$ \\
\hline Attention & $\begin{array}{l}\text { Attention refers to the ability to focus the awareness on a specific } \\
\text { stimulus or stimuli, allowing for a limited but more intense appraisal } \\
\text { of the current experience. }\end{array}$ & $\begin{array}{l}\text { Langer, 2000; Kabat-Zinn, } \\
\text { 1978; Feldman, G.,Hayes, A., } \\
\text { Kumar, S., Greeson, J., \& } \\
\text { Laurenceau, J. P. (2007); } \\
\text { Walach, H., Buchheld, N., } \\
\text { Butternmuller, V., } \\
\text { Kleinknecht, N., Schmidt, S. } \\
\text { (2006); Tanay, G., Bernstein, } \\
\text { A. (2013) }\end{array}$ \\
\hline $\begin{array}{l}\text { Awareness, } \\
\text { Acting with } \\
\text { awareness, } \\
\text { Self- } \\
\text { Awareness, }\end{array}$ & $\begin{array}{l}\text { Self-Awareness and others. Deliberate way to sustain attention and } \\
\text { expand the perception of the body and environment. And It is what } \\
\text { allows us to notice things around us without focusing exclusively on } \\
\text { them. }\end{array}$ & $\begin{array}{l}\text { Langer, 2000; Kabat-Zinn, } \\
\text { 2003; Vande et al. 2015, } \\
\text { Djikic, 2014, Nhat Hanh, } \\
\text { Gunaratana, Kabat-Zinn, } \\
\text { 2003; Ostafin et al. 2015; Au } \\
2013 \text { and 2015; Feldman, } \\
\text { G.,Hayes, A., Kumar, S., } \\
\text { Greeson, J., \& Laurenceau, J. } \\
\text { P. (2007); Walach, H., } \\
\text { Buchheld, N., Butternmuller, } \\
\text { V., Kleinknecht, N., Schmidt, } \\
\text { S. (2006); Cardaciotto, L. et al } \\
\text { (2008); Bergomi, C; } \\
\text { Tschacher, W. \&Kupper, Z. } \\
\text { (2014); Cardatiotto, L. et al } \\
\text { (2008); Tanay, G., Bernstein, } \\
\text { A. (2013) Baer, R.A., Smith, G. } \\
\text { t., Hopkins, J., Krietemeyer, J., } \\
\text { \& Toney, L. (2006) }\end{array}$ \\
\hline $\begin{array}{l}\text { Decentring / } \\
\text { Non-reacting }\end{array}$ & $\begin{array}{l}\text { A shift in one's cognitive perspective known as decentering or } \\
\text { disidentification represents a different way to observe the thoughts } \\
\text { and consequently the attitude without react to them. }\end{array}$ & $\begin{array}{l}\text { Lau, M.A. et al (2006): } \\
\text { Bergomi, C; Tschacher, W. } \\
\text { \&Kupper, Z. (2014); } \\
\text { Baer, R.A., Smith, G. t., } \\
\text { Hopkins, J., Krietemeyer, J., \& } \\
\text { Toney, L. (2006) }\end{array}$ \\
\hline Describing & $\begin{array}{l}\text { Describing. It is an ability to describe or labelling emotion as } \\
\text { "worrying about my job", "I'm sad", or even contextualize the } \\
\text { feeling, "Ah, here is anger". This type of describing is done }\end{array}$ & $\begin{array}{l}\text { Baer, R.A., Smith, G. t., } \\
\text { Hopkins, J., Krietemeyer, J., \& } \\
\text { Toney, L. (2006); }\end{array}$ \\
\hline
\end{tabular}




\begin{tabular}{|c|c|c|}
\hline & $\begin{array}{l}\text { nonjudgmentally and without conceptual analysis. That is, } \\
\text { participants are encouraged to refrain from judgments (e.g., "it's } \\
\text { stupid to think this way") and from speculations about the origins of } \\
\text { these patterns. Instead, they are instructed to label them briefly and } \\
\text { continue attending to the present moment. }\end{array}$ & $\begin{array}{l}\text { Baer, R.A., Smith, G. T., \& } \\
\text { Allen, K. B. (2004) }\end{array}$ \\
\hline Engagement & $\begin{array}{l}\text { Mindfulness training induce the practitioner to actively attend to } \\
\text { the details of their immediate experience of action engagement as } \\
\text { they emerge. }\end{array}$ & $\begin{array}{l}\text { Langer, 2000; Nhat Hanh } \\
\text { 1976; Rojas et al. 2015; } \\
\text { Yeganeh \& Kolb, } 2009\end{array}$ \\
\hline Empathy & $\begin{array}{l}\text { Empathy is used to bring attention and awareness to user's needs } \\
\text { and others. Empathy leads to interact and engage with others. }\end{array}$ & $\begin{array}{l}\text { Rojas et al 2016,2017, Kabat- } \\
\text { Zinn 1978, Au,2013; Shapiro } \\
\text { et al. 1998, Krasner et al. } \\
\text { 2009; Au } 2013 \text { and 2015; }\end{array}$ \\
\hline Focus & $\begin{array}{l}\text { Maintaining focus on moment-to-moment, every action. Refers to } \\
\text { our ability to focus the awareness on a specific stimulus or stimuli, } \\
\text { allowing for a limited but more intense appraisal of our current } \\
\text { experience. }\end{array}$ & $\begin{array}{l}\text { Langer, 2000; Kabat-Zinn, } \\
\text { 1978; Yeganeh \& Kolb, 2009; } \\
\text { Au } 2013 \text { and 2015, Feldman, } \\
\text { G.,Hayes, A., Kumar, S., } \\
\text { Greeson, J., \& Laurenceau, J. } \\
\text { P. (2007) }\end{array}$ \\
\hline $\begin{array}{l}\text { Non- } \\
\text { judgement }\end{array}$ & $\begin{array}{l}\text { Most mindfulness conceptualizations emphasize the importance of } \\
\text { acceptance or being non-judgmental. That means not labelling as } \\
\text { "good" or "bad;" instead, the acceptance/non-judgment piece of } \\
\text { mindfulness encourages a neutral perspective and an acceptance of } \\
\text { thoughts or feeling without criticism them. }\end{array}$ & $\begin{array}{l}\text { Kabat-Zinn, 1978; Bergomi, C; } \\
\text { Tschacher, W. \&Kupper, Z. } \\
\text { (2014); Walach, H. et al } \\
\text { (2006), Baer, R. A., Smith, G. } \\
\text { T., Hopkins, J., Krietemeyer, J., } \\
\text { \& Toney, L. (2006); Bergomi, } \\
\text { C; Tschacher, W. \&Kupper, Z. } \\
\text { (2014); Baer, R.A., Smith, G. } \\
\text { T., \& Allen, K. B. (2004) }\end{array}$ \\
\hline $\begin{array}{l}\text { Novelty/ } \\
* \text { Curiosity }\end{array}$ & $\begin{array}{l}\text { See the facts with novelty eyes, in a different attitude. Be creative. } \\
\text { To like to learn new tasks. }\end{array}$ & $\begin{array}{l}\text { Kabat-Zinn, 1994; Langer, } \\
\text { 2000, Shapiro et al. 2006; } \\
\text { Rosenberg } 2015 . \\
\text { *Tanay, G., Bernstein, A. } \\
\text { (2013); Walach, H., Buchheld, } \\
\text { N., Butternmuller, V., } \\
\text { Kleinknecht, N., Schmidt, S. } \\
\text { (2006); Lau, M.A. et al (2006) }\end{array}$ \\
\hline Observing & $\begin{array}{l}\text { It Is the task to observe, notice, or attending to a variety of stimuli, } \\
\text { including internal phenomena, such as bodily sensations, cognitions, } \\
\text { and emotions, and external phenomena, such as sounds and smells. } \\
\text { People are encouraged to pay careful attention to elements such as } \\
\text { the location, intensity, duration of sensations, and the pitch, } \\
\text { volume, and tone quality of sounds. }\end{array}$ & $\begin{array}{l}\text { Baer, R.A., Smith, G. t., } \\
\text { Hopkins, J., Krietemeyer, J., \& } \\
\text { Toney, L. (2006); } \\
\text { Baer, R.A., Smith, G. T., \& } \\
\text { Allen, K. B. (2004) }\end{array}$ \\
\hline Openness & $\begin{array}{l}\text { Maintain an orientation in the present, an alertness to distinctions, } \\
\text { a sensitivity to different contexts, and an openness to novelty and } \\
\text { information. }\end{array}$ & $\begin{array}{l}\text { Langer, 1990; Walsh and } \\
\text { Shapiro, 2006; Yeganeh \& } \\
\text { Kolb 2009; Au } 2013 \text { and 2015; } \\
\text { Bishop et al. 2004; Walach, H., } \\
\text { Buchheld, N., Butternmuller, } \\
\text { V., Kleinknecht, N., Schmidt, } \\
\text { S. (2006); Lau, M.A. et al } \\
\text { (2006) }\end{array}$ \\
\hline Purpose & $\begin{array}{l}\text { Purpose for mindfulness state, refers to put intention and focus to } \\
\text { thought in every given activity. }\end{array}$ & $\begin{array}{l}\text { Kabat-Zinn, 1978; Au } 2013 \\
\text { and } 2015\end{array}$ \\
\hline
\end{tabular}


Uma visão geral dessas avaliações por autorrelato é categorizada na matriz abaixo, dividida pelo nome do questionário de autorrelato, os mecanismos categorizados de mindfulness e os critérios de ferramenta: se o questionário é relacionado a um traço, um estado de mindfulness, se é usado para abordagens meditativas ou sócio-cognitivas, para identificar as necessidades do usuário como uma entrevista que pode ser usada em terceira pessoa ou apenas autorrelato. Se esses questionários foram usados para avaliação do produto ou para medir a experiência do usuário em um nível de produto. $\mathrm{E}$, se o mindfulness foi usado em uma investigação de curto prazo como um estado ou se foi usado como uma avaliação de longo prazo para determinar o traço do paciente ao longo de determinado período. Os blocos brancos indicam o ponto de correspondência da categorização.

Image 2: Visão geral das principais avaliações de autorrelato categorizadas nos mecanismos de Mindfulness e nos objetivos das ferramentas.

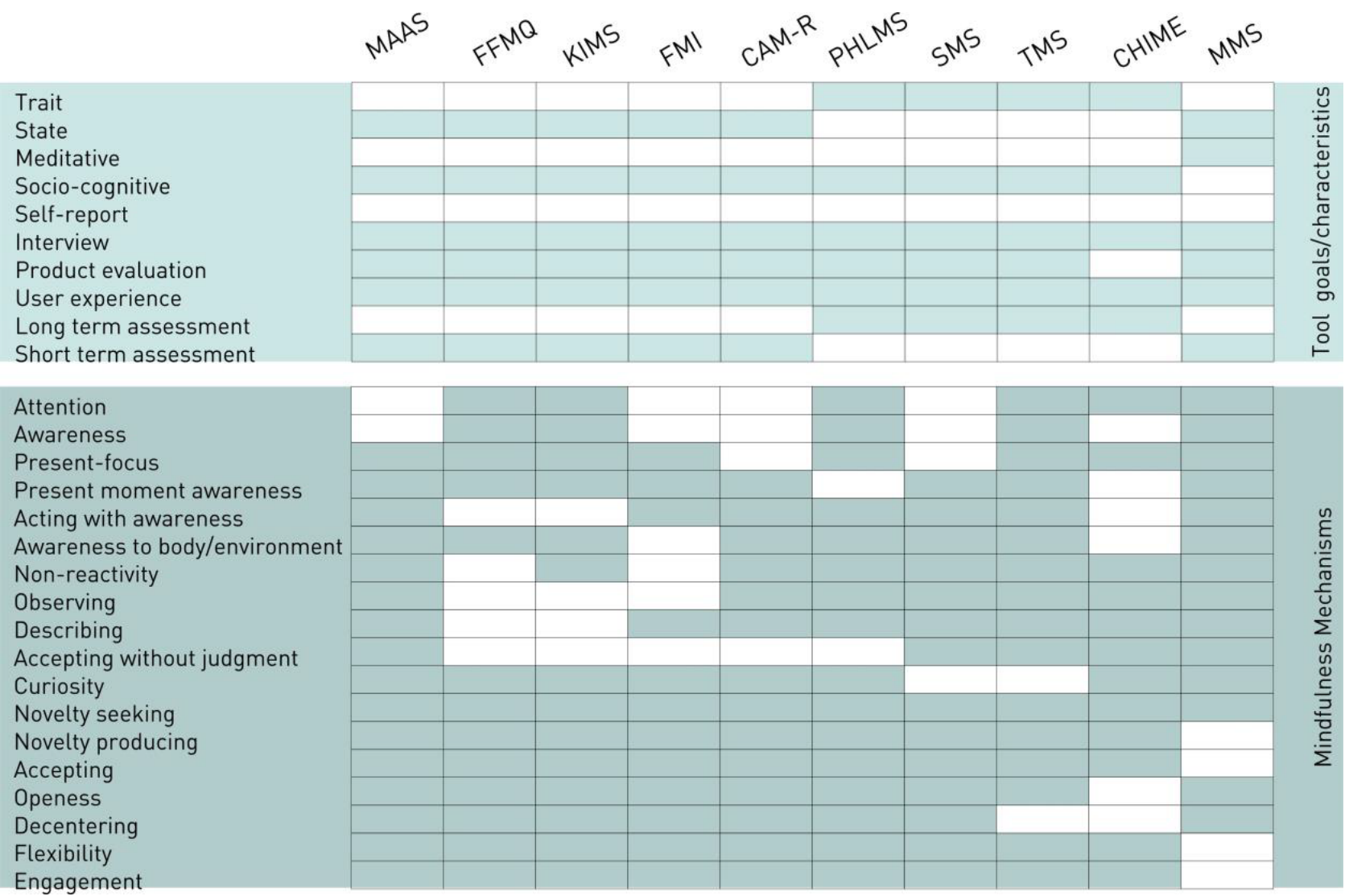

Com base na descrição da autoavaliação e na visualização de suas diferentes abordagens e objetivos, é necessário discutir sobre a seleção das declarações de avaliação do Mindful Design.

É interessante observar que apenas uma autoavaliação foi utilizada com o propósito de avaliar o design por meio da classificação de tecnologias interativas, o CHIME (SLIWINSKI et al., 2017). Sliwinski et al (2017), em seu estudo, apresentaram abordagens de tecnologia interativa para melhorar as qualidades de mindfulness e discutem suas implicações em jogos, aplicativos para smartphones e software de computador. Nesse caso de estudo, o questionário de autorrelato não é adequado porque não é focado nos mecanismos de atenção sócio-cognitivos, mas nos meditativos. 
A partir dos objetivos de autoavaliação, importantes para uma análise do Mindful Design, apenas um dos questionários considera os mecanismos sócio-cognitivos: engajamento, busca e produção de novidades, flexibilidade. A curiosidade poderia ser incluída na busca por novidades, que descreve esse mecanismo de maneira muito semelhante. Por exemplo: Curiosidade em TMS "Eu estava curioso sobre o que eu poderia aprender sobre mim mesmo, tomando conhecimento de como eu reajo a certos pensamentos, sentimentos ou sensações" ou/e buscando novidades em LMS "Eu tento pensar em novas maneiras de fazer as coisas". Mas, o autorrelato de TMS tem metas de avaliação diferentes. Enquanto a de TMS considera mindfulness como um estado, LMS considera como traço.

Outro motivo para não usar esses questionários é porque eles têm uma forte influência na atenção meditativa, não sendo muito genéricos em suas declarações sobre a curiosidade. Por essas razões, o TMS não poderia ser aplicado como ferramenta de avaliação do Mindful Design.

O mecanismo de Minfulness é abordado por oito dos dez comentários da autoavaliação. E, mesmo os dois restantes têm alguma descrição sobre a consciência em suas declarações, por exemplo: TMS "Eu estava ciente dos meus pensamentos e sentimentos sem me identificar mais com eles" ou (MMS) "Eu raramente estou ciente das mudanças". Esse fato nos fez manter a consciência como um mecanismo de mindfulness sócio-cognitivo para produtos de design, mesmo quando essa característica não é explícita na autoavaliação sócio-cognitiva em si.

A atenção tem o mesmo valor que a conscientização para aplicação no design de produtos. Porém, ela tem sido aplicada com outras dimensões de mindfulness como "observar" (CHIME) e "agir com consciência" (FFMQ). Conforme a explicação sobre a dimensão "curiosidade", o CHIME não tem os mesmos objetivos de avaliação e tem um forte enfoque na mensuração do aumento da atenção plena pela prática da meditação, diferentemente da avaliação sócio-cognitiva do LMS.

A partir dessas análises, o questionário de autorrelato de Mindfulness escolhido foi LMS (a versão revisada do MMS) e MAAS (consciência e atenção). O FFMQ e o KIMS poderiam ser considerados, por terem objetivos de avaliação semelhantes, declarações similares e genéricas para analisar as dimensões de atenção e conscientização. Mas, para a primeira avaliação, foram usados o LMS e o MAAS para não dividir desnecessariamente as configurações do construto.

\section{Desenvolvimento de uma ferramenta de avaliação de Mindful Design}

O método transferido do campo da psicologia, o autorrelato psicométrico para a mensuração da atenção plena, tem sido selecionado e adaptado ao contexto de desenvolvimento de produtos, com o intuito de medir o nível de Mindfulness sócio-cognitivo que os produtos podem alcançar.

Para projetar uma ferramenta de avaliação padrão, algumas regras foram consideradas. Segundo Mertens (2005), há quatro tipos principais de validade usados para selecionar um método de avaliação:

- Validade metodológica: validade de conteúdo, validade relacionada ao critério, validade de construto e generalização;

- Validade interpessoal: influências pessoais, crenças e valores;

- Validade consequencial: catalisador de mudança, efeitos não intencionais;

- Validade multicultural: tempo, sofisticação cultural e prevenção da complacência 
arrogante.

Considerando que as autoavaliações já são todas metodologicamente consideradas, o desenvolvimento dessa ferramenta de avaliação é dividido em três etapas:

Fase 1 - Quais afirmações podem ser selecionadas para medir o nível de atenção plena?

Fase 2 - As declarações são confiáveis, fáceis de entender e aplicáveis à interação do usuário com o produto? Pode ser facilmente respondido por seus objetivos?

Fase 3 - Os níveis de Mindfulness podem ser identificados entre diferentes soluções de produto?

\subsection{Fase 1 - Seleção de declarações}

A ideia de desenvolver uma ferramenta de avaliação para o Mindful Design (design consciente) preencheu um requisito muito importante do processo de desenvolvimento do produto: estruturar os objetivos do projeto e a autoavaliação dos conceitos.

Usando estratégias para reconhecer os objetivos iniciais do projeto é possível visualizar as prioridades estabelecidas e focar na solução do problema do projeto.

Como forma de avaliar os conceitos desenvolvidos de Mindful Design, propusemos uma ferramenta de avaliação composta pelos mecanismos considerados importantes para o estado sócio-cognitivo da atenção plena. Na tabela abaixo é possível identificar os mecanismos, bem como a proveniência dos depoimentos selecionados nos 10 questionários de autorrelato mais utilizados de acordo com a literatura.

Nesta tabela foram coletadas afirmações referentes aos seguintes mecanismos: busca de novidade e produção de novidade, engajamento e flexibilidade, bem como consciência e atenção. Importante para a compreensão da atenção consciente sócio-cognitiva.

Tabela 2 Declarações de autorrelato sócio-cognitivo de Mindfulness.

\begin{tabular}{|c|c|c|}
\hline \multicolumn{3}{|c|}{ Mindfulness dimensions } \\
\hline Acceptance & $\begin{array}{l}\text { Some authors mean acceptance as non-judgment. Some author split } \\
\text { these mechanisms in two different constructs. }\end{array}$ & $\begin{array}{l}\text { Bergomi, C; Tschacher, W. } \\
\text { \&Kupper, Z. (2014); } \\
\text { Cardatiotto, L. et al (2008); } \\
\text { Feldman, G. et al (2007); Baer, } \\
\text { R. A., Smith G. T., \& Allen, KB. } \\
\text { (2004) }\end{array}$ \\
\hline Attention & $\begin{array}{l}\text { Attention refers to the ability to focus the awareness on a specific } \\
\text { stimulus or stimuli, allowing for a limited but more intense appraisal } \\
\text { of the current experience. }\end{array}$ & $\begin{array}{l}\text { Langer, 2000; Kabat-Zinn, } \\
\text { 1978; Feldman, G.,Hayes, A., } \\
\text { Kumar, S., Greeson, J., \& } \\
\text { Laurenceau, J. P. (2007); } \\
\text { Walach, H., Buchheld, N., } \\
\text { Butternmuller, V., } \\
\text { Kleinknecht, N., Schmidt, S. } \\
\text { (2006); Tanay, G., Bernstein, } \\
\text { A. (2013) }\end{array}$ \\
\hline $\begin{array}{l}\text { Awareness, } \\
\text { Acting with } \\
\text { awareness, }\end{array}$ & $\begin{array}{l}\text { Self-Awareness and others. Deliberate way to sustain attention and } \\
\text { expand the perception of the body and environment. And It is what }\end{array}$ & $\begin{array}{l}\text { Langer, 2000; Kabat-Zinn, } \\
\text { 2003; Vande et al. 2015, } \\
\text { Djikic, 2014, Nhat Hanh, }\end{array}$ \\
\hline
\end{tabular}




\begin{tabular}{|c|c|c|}
\hline $\begin{array}{l}\text { Self- } \\
\text { Awareness, }\end{array}$ & $\begin{array}{l}\text { allows us to notice things around us without focusing exclusively on } \\
\text { them. }\end{array}$ & $\begin{array}{l}\text { Gunaratana, Kabat-Zinn, } \\
\text { 2003; Ostafin et al. 2015; Au } \\
2013 \text { and 2015; Feldman, } \\
\text { G.,Hayes, A., Kumar, S., } \\
\text { Greeson, J., \& Laurenceau, J. } \\
\text { P. (2007); Walach, H., } \\
\text { Buchheld, N., Butternmuller, } \\
\text { V., Kleinknecht, N., Schmidt, } \\
\text { S. (2006); Cardaciotto, L. et al } \\
\text { (2008); Bergomi, C; } \\
\text { Tschacher, W. \&Kupper, Z. } \\
\text { (2014); Cardatiotto, L. et al } \\
\text { (2008); Tanay, G., Bernstein, } \\
\text { A. (2013) Baer, R.A., Smith, G. } \\
\text { t., Hopkins, J., Krietemeyer, J., } \\
\text { \& Toney, L. (2006) }\end{array}$ \\
\hline $\begin{array}{l}\text { Decentring / } \\
\text { Non-reacting }\end{array}$ & $\begin{array}{l}\text { A shift in one's cognitive perspective known as decentering or } \\
\text { disidentification represents a different way to observe the thoughts } \\
\text { and consequently the attitude without react to them. }\end{array}$ & $\begin{array}{l}\text { Lau, M.A. et al (2006): } \\
\text { Bergomi, C; Tschacher, W. } \\
\text { \&Kupper, Z. (2014); } \\
\text { Baer, R.A., Smith, G. t., } \\
\text { Hopkins, J., Krietemeyer, J., \& } \\
\text { Toney, L. (2006) }\end{array}$ \\
\hline Describing & $\begin{array}{l}\text { Describing. It is an ability to describe or labelling emotion as } \\
\text { "worrying about my job", "I'm sad", or even contextualize the } \\
\text { feeling, "Ah, here is anger". This type of describing is done } \\
\text { nonjudgmentally and without conceptual analysis. That is, } \\
\text { participants are encouraged to refrain from judgments (e.g., "it's } \\
\text { stupid to think this way") and from speculations about the origins of } \\
\text { these patterns. Instead, they are instructed to label them briefly and } \\
\text { continue attending to the present moment. }\end{array}$ & $\begin{array}{l}\text { Baer, R.A., Smith, G. t., } \\
\text { Hopkins, J., Krietemeyer, J., \& } \\
\text { Toney, L. (2006); } \\
\text { Baer, R.A., Smith, G. T., \& } \\
\text { Allen, K. B. (2004) }\end{array}$ \\
\hline Engagement & $\begin{array}{l}\text { Mindfulness training induce the practitioner to actively attend to } \\
\text { the details of their immediate experience of action engagement as } \\
\text { they emerge. }\end{array}$ & $\begin{array}{l}\text { Langer, 2000; Nhat Hanh } \\
\text { 1976; Rojas et al. 2015; } \\
\text { Yeganeh \& Kolb, } 2009\end{array}$ \\
\hline Empathy & $\begin{array}{l}\text { Empathy is used to bring attention and awareness to user's needs } \\
\text { and others. Empathy leads to interact and engage with others. }\end{array}$ & $\begin{array}{l}\text { Rojas et al 2016,2017, Kabat- } \\
\text { Zinn 1978, Au,2013; Shapiro } \\
\text { et al. 1998, Krasner et al. } \\
\text { 2009; Au } 2013 \text { and 2015; }\end{array}$ \\
\hline Focus & $\begin{array}{l}\text { Maintaining focus on moment-to-moment, every action. Refers to } \\
\text { our ability to focus the awareness on a specific stimulus or stimuli, } \\
\text { allowing for a limited but more intense appraisal of our current } \\
\text { experience. }\end{array}$ & $\begin{array}{l}\text { Langer, 2000; Kabat-Zinn, } \\
\text { 1978; Yeganeh \& Kolb, 2009; } \\
\text { Au } 2013 \text { and 2015, Feldman, } \\
\text { G.,Hayes, A., Kumar, S., } \\
\text { Greeson, J., \& Laurenceau, J. } \\
\text { P. (2007) }\end{array}$ \\
\hline $\begin{array}{l}\text { Non- } \\
\text { judgement }\end{array}$ & $\begin{array}{l}\text { Most mindfulness conceptualizations emphasize the importance of } \\
\text { acceptance or being non-judgmental. That means not labelling as } \\
\text { "good" or "bad;" instead, the acceptance/non-judgment piece of } \\
\text { mindfulness encourages a neutral perspective and an acceptance of } \\
\text { thoughts or feeling without criticism them. }\end{array}$ & $\begin{array}{l}\text { Kabat-Zinn, 1978; Bergomi, C; } \\
\text { Tschacher, W. \&Kupper, Z. } \\
\text { (2014); Walach, H. et al } \\
\text { (2006), Baer, R. A., Smith, G. } \\
\text { T., Hopkins, J., Krietemeyer, J., } \\
\text { \& Toney, L. (2006); Bergomi, } \\
\text { C; Tschacher, W. \&Kupper, Z. } \\
\text { (2014); Baer, R.A., Smith, G. } \\
\text { T., \& Allen, K. B. (2004) }\end{array}$ \\
\hline
\end{tabular}




\begin{tabular}{|c|c|c|}
\hline $\begin{array}{l}\text { Novelty/ } \\
* \text { Curiosity }\end{array}$ & $\begin{array}{l}\text { See the facts with novelty eyes, in a different attitude. Be creative. } \\
\text { To like to learn new tasks. }\end{array}$ & $\begin{array}{l}\text { Kabat-Zinn, 1994; Langer, } \\
\text { 2000, Shapiro et al. 2006; } \\
\text { Rosenberg } 2015 . \\
\text { *Tanay, G., Bernstein, A. } \\
\text { (2013); Walach, H., Buchheld, } \\
\text { N., Butternmuller, V., } \\
\text { Kleinknecht, N., Schmidt, S. } \\
\text { (2006); Lau, M.A. et al (2006) }\end{array}$ \\
\hline Observing & $\begin{array}{l}\text { It Is the task to observe, notice, or attending to a variety of stimuli, } \\
\text { including internal phenomena, such as bodily sensations, cognitions, } \\
\text { and emotions, and external phenomena, such as sounds and smells. } \\
\text { People are encouraged to pay careful attention to elements such as } \\
\text { the location, intensity, duration of sensations, and the pitch, } \\
\text { volume, and tone quality of sounds. }\end{array}$ & $\begin{array}{l}\text { Baer, R.A., Smith, G. t., } \\
\text { Hopkins, J., Krietemeyer, J., \& } \\
\text { Toney, L. (2006); } \\
\text { Baer, R.A., Smith, G. T., \& } \\
\text { Allen, K. B. (2004) }\end{array}$ \\
\hline Openness & $\begin{array}{l}\text { Maintain an orientation in the present, an alertness to distinctions, } \\
\text { a sensitivity to different contexts, and an openness to novelty and } \\
\text { information. }\end{array}$ & $\begin{array}{l}\text { Langer, 1990; Walsh and } \\
\text { Shapiro, 2006; Yeganeh \& } \\
\text { Kolb 2009; Au } 2013 \text { and 2015; } \\
\text { Bishop et al. 2004; Walach, H., } \\
\text { Buchheld, N., Butternmuller, } \\
\text { V., Kleinknecht, N., Schmidt, } \\
\text { S. (2006); Lau, M.A. et al } \\
\text { (2006) }\end{array}$ \\
\hline Purpose & $\begin{array}{l}\text { Purpose for mindfulness state, refers to put intention and focus to } \\
\text { thought in every given activity. }\end{array}$ & $\begin{array}{l}\text { Kabat-Zinn, 1978; Au } 2013 \\
\text { and } 2015\end{array}$ \\
\hline
\end{tabular}

\subsection{Fase 2A: Fiabilidade das declarações, facilidade de compreensão e aplicabilidade}

A seleção das declarações foi baseada nos seguintes critérios:

- Homogeneidade e declarações gerais;

- Mesma construção: usar as declarações do mesmo conjunto de questionário;

- Bem estabelecido: usar declarações de um conjunto de questionário bem estabelecido;

- Fácil compreensão: se houver sobreposição de significados entre as afirmações do questionário, usar o mais fácil de entender;

- Aplicável à interação do produto: selecionar apenas as declarações que são possíveis de imaginar na interação com os produtos.

Com base nesses critérios, o primeiro esboço da ferramenta de avaliação foi uma combinação da autoavaliação de LMS de Pirson et al. (2012) somado ao mecanismo de flexibilidade mencionado em MMS de Bodner e Langer (2001) e MAAS de Brown e Ryan (2003). Foi uma escala de 32 itens envolvendo os mecanismos de busca de novidades, produção de novidades, engajamento, flexibilidade, consciência e atenção.

A pergunta a ser respondida foi: "Como o usuário responderia às seguintes questões ao usar / interagir com o produto?" A escala usada para medi-lo foi de "concordo totalmente" a "discordo totalmente", dependendo de quão precisamente a afirmação se encaixa na situação. Também foi possível marcar a opção "NA", se não aplicável. Veja o exemplo da ferramenta de avaliação abaixo. 
Imagem 2 Avaliação do produto de Mindful Design

\begin{tabular}{|c|c|c|c|c|}
\hline & $\begin{array}{l}\text { How would the user answer the following questions when } \\
\text { using/interacting with the product? }\end{array}$ & $\begin{array}{l}\text { Strongly } \\
\text { agree }\end{array}$ & $\begin{array}{l}\text { Strongly } \\
\text { disagree }\end{array}$ & NA \\
\hline 1 & $\begin{array}{l}\text { I find it difficult to stay focused on what's happening in } \\
\text { the present. (AT) }\end{array}$ & & 0 & 0 \\
\hline 2 & $\begin{array}{l}\text { I break or spill things because of carelessness, not paying } \\
\text { attention, or thinking of something else. (AT) }\end{array}$ & & $\cap$ & O \\
\hline 3 & $\begin{array}{l}\text { I tend not to notice feelings of physical tension or } \\
\text { discomfort until they really grab my attention. (AT) }\end{array}$ & & & O \\
\hline 4 & $\begin{array}{l}\text { It's seems I am "running on automatic “, without much } \\
\text { awareness of what I'm doing. (AT) }\end{array}$ & & & O \\
\hline 5 & $\begin{array}{l}\text { I find myself preoccupied with the future or the past. } \\
\text { (AT) }\end{array}$ & & & O \\
\hline 6 & I find myself doing things without paying attention. (AT) & & & O \\
\hline 7 & $\begin{array}{l}\text { I tend to walk quickly to get where I'm going without } \\
\text { paying attention to what I experience along the way. (AT) }\end{array}$ & & & D \\
\hline 8 & I seldom notice what other people are up to. (E) & & & O \\
\hline 9 & I avoid thought provoking conversations. (E) & & & O \\
\hline 10 & I am rarely aware of changes. $(E)$ & & & O \\
\hline 11 & I am rarely alert to new developments. (E) & & & O \\
\hline 12 & I am very curious. (NS) & & & O \\
\hline 13 & I like to investigate things. (NS) & & & O \\
\hline 14 & I try to think of new ways of doing things. (NS) & & & D \\
\hline 15 & I like to be challenged intellectually. (NS) & & & O \\
\hline 16 & I like to figure out how things work. (NS) & & & b \\
\hline 17 & I generate few novel ideas. (NP) & & & b \\
\hline 18 & I make many novel contributions. (NP) & & & O \\
\hline 19 & I am very creative. (NP) & & & O \\
\hline 20 & I find it easy to create new and effective ideas. (NP) & & & O \\
\hline 21 & I am not an original thinker. (NP) & & & O \\
\hline 22 & I am always open to new ways of doing things. (F) & & & \\
\hline 23 & I stay with the old tried and true ways of doing things. (F) & & & \\
\hline 24 & $\begin{array}{l}\text { I can behave in many different ways for a given situation. } \\
\text { (F) }\end{array}$ & & & \\
\hline 25 & $\begin{array}{l}\text { I have an open mind about everything, even things that } \\
\text { challenge my core beliefs. (F) }\end{array}$ & & & \\
\hline 26 & $\begin{array}{l}\text { I find myself listening to someone with one ear, doing } \\
\text { something else at the same time. (A) }\end{array}$ & & & \\
\hline 27 & $\begin{array}{l}\text { I do jobs or tasks automatically, without being of what } \\
\text { I'm doing (A) }\end{array}$ & & & \\
\hline 28 & $\begin{array}{l}\text { I get so focused on the goal I want to achieve that I lose } \\
\text { touch with what I'm doing right now to get there (A) }\end{array}$ & & & 0 \\
\hline 29 & I snack without being aware that I'm eating $(A)$ & & O & \\
\hline 30 & $\begin{array}{l}\text { I could be experiencing some emotion and not be } \\
\text { conscious of it until sometime later (A) }\end{array}$ & & & \\
\hline 31 & $\begin{array}{l}\text { I do jobs or tasks automatically, without being aware of } \\
\text { what i'm doing. (A) }\end{array}$ & & 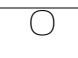 & \\
\hline 32 & $\begin{array}{l}\text { I rush through activities without being really attentive to } \\
\text { them. (A) }\end{array}$ & & O & D \\
\hline
\end{tabular}

A escala foi testada usando três diferentes conceitos de produtos conscientes, desenvolvidos pelos alunos, em três cenários diferentes com pessoas que sofrem de demência. Três outros especialistas em design deveriam visualizar e imaginar os cenários com os conceitos de produtos propostos e responder à declaração como eles os entenderam. Depois disso, eles discutiram sobre as declarações.

Algumas das considerações discutidas foram de que era difícil aplicar essas declarações usando esse formato, devido à escala Likert de "concordo totalmente" a "discordo totalmente". Talvez uma escala que fosse de "tornar-se melhor" a "tornar-se pior" possa ser usada para entender se o produto ou conceito seria capaz de melhorar ou não na obtenção do estado de mindfulness (atenção plena).

Declarações como "Eu lancho sem estar ciente de que estou comendo", na opinião deles, representam uma tarefa muito específica e poderiam ser agrupadas em qualquer uma das opções da escala em relação aos exemplos de conceitos de produto. Por esse motivo, uma segunda versão 
do questionário foi elaborada para avaliar esses ajustes.

\subsection{Fase 2B: Fiabilidade das declarações, facilidade de compreensão e aplicabilidade - aperfeiçoamento}

A segunda versão do questionário de avaliação teve os seguintes ajustes em comparação com o primeiro:

Escala: a escala indica de "tornar-se pior" para "tornar-se melhor" dependendo de como a interação usuário-produto melhora/piora a condição de atenção do usuário. Uma escala de 29 itens projetada para medir a atenção plena durante a interação com um produto, em relação a seus construtos como: engajamento, busca de novidades, produção de novidades, flexibilidade, consciência e atenção ocorridos no momento da interação do usuário.

Conjunto de declarações: Esta escala de avaliação foi extraída de 3 fontes validadas: MAAS (Mindful Attention Awareness Scale) de Brown e Ryan (2003) sobre atenção; FFMQ (Five Facet Mindfulness Questionnaire) de Baer et al. (2006) sobre conscientização; e, um autorrelato validado para o LMS sócio-cognitivo (Langer Mindfulness Scale) de Pirson et al. (2012) relacionado ao engajamento, procura e produção de novidades (incluindo flexibilidade).

Flexibilidade: As declarações de flexibilidade já foram avaliadas e estatisticamente comprovadas em estudos realizados por Pirson et al. (2012). Não há significância em comparação com os outros mecanismos de mindfulness sócio-cognitivos. Por esse motivo, essas declarações foram removidas na nova versão do questionário.

Não aplicável: não aplicável foi removido. Em vez disso, o ponto central indica uma opinião neutra, no caso de a declaração não se modificar com a interação.

De acordo com esses ajustes, segue o novo design da escala para avaliar produtos de design consciente: 
Imagem 3 Avaliação do produto de Mindful Design atualizado

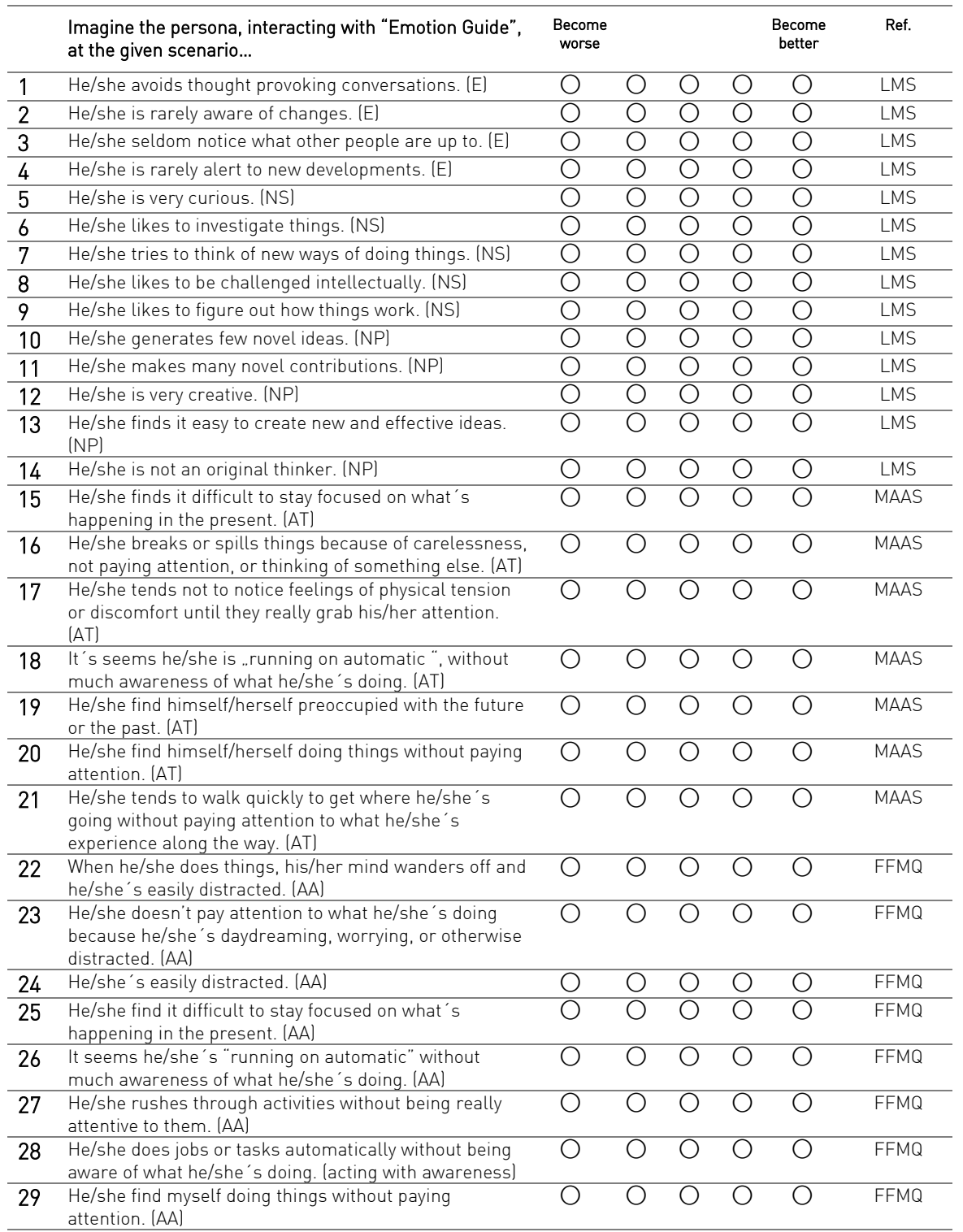

* Os resultados e análises das ferramentas de avaliação do Mindful Design ainda estão em andamento e serão apresentados no artigo de revisão.

\section{Discussão}

A discussão sobre os benefícios do treinamento em atenção plena para pessoas com menor desempenho cognitivo, pacientes com transtornos mentais ou pessoas que pretendem aplicar técnicas de mindfulness para melhorar sua qualidade de vida têm sido amplamente pesquisadas. Por essa razão, o aumento da necessidade de investigar o impacto dessas práticas a curto e longo prazo também cresceu. Há muitas evidências de que a atenção plena pode melhorar o modo como as pessoas enfrentam desafios em suas vidas, apenas trabalhando ativamente em sua consciência interna e social. Praticando mindfulness, meditando ou deliberadamente mantendo sua atenção em suas atividades, ativa o senso de comportamento de autorregulação e, consequentemente, seu bem-estar subjetivo. 
Sendo assim, é importante investigar mais profundamente o uso prático da atenção plena para aplicação no design. Os produtos influenciam diariamente nosso comportamento, consciente e inconscientemente. Empreender os mecanismos de mindfulness em produtos de uso cotidiano podem transferi-los para o comportamento do usuário.

A intenção de desenvolver uma ferramenta de avaliação para os produtos Mindful Design atinge as qualidades e mecanismos da atenção plena nos dois sentidos, para que o usuário atinja esse estado de espírito e para que os designers se tornem empáticos e tenham uma melhor percepção do que o usuário precisa.

Essa ferramenta de avaliação pode ser uma ajuda essencial a ser aplicada no início do processo de design, na idealização do projeto, para identificar e descrever quais características de atenção plena do produto estão sendo bem-sucedidas e quais podem ser melhoradas.

Finalmente, um workshop com especialistas será apresentado para testar a eficiência da ferramenta de avaliação ao apoiar projetos de design para atenção plena.

\section{Referências}

ACKERMAN, Courtney (2017): 11 Mindfulness Questionnaires, Scales \& Assessments For Measuring. positive psychology. Available online at https://positivepsychologyprogram.com/mindfulnessquestionnaires-scales-assessments-awareness/\#challenges-measuring-mindfulness, checked on 9/20/2017.

AKAMA, Yoko; Light, Ann; Bowen, Simon: Mindfulness and Technology. In Oli Mival, Michael Smyth, Peter Dalsgaard (Eds.): the 2017 Conference. Edinburgh, United Kingdom, pp. 345-355.

AU, Irene (2013): Mindful Design: What the UX World Can Learn from Yoga User Experience Magazine. Edited by UX Perspectives. Available online at http://uxpamagazine.org/mindful-design/, checked on 8/17/2017.

AU, Irene (2015): Mindfulness Practices For Design - Design Your Life - Medium. Available online at https://medium.com/design-your-life/mindfulness-practices-for-design-9f8b7f1af047, checked on 8/14/2017.

BAER, Ruth A.; Smith, Gregory T.; Lykins, Emily; Button, Daniel; Krietemeyer, Jennifer; Sauer, Shannon et al. (2008): Construct validity of the five facet mindfulness questionnaire in meditating and nonmeditating samples. In Assessment 15 (3), pp. 329-342. DOI: 10.1177/1073191107313003.

BERGOMI, Claudia; Tschacher, Wolfgang; Kupper, Zeno (2015): Meditation Practice and SelfReported Mindfulness: a Cross-Sectional Investigation of Meditators and Non-Meditators Using the Comprehensive Inventory of Mindfulness Experiences (CHIME). In Mindfulness 6 (6), pp. 14111421. DOI: $10.1007 / \mathrm{s} 12671-015-0415-6$.

BODNER T., Langer E. (2001). “Individual differences in mindfulness: the mindfulness/mindlessness scale," in Poster Presented at the 13th Annual American Psychological Society Conference Toronto.

CARDACIOTTO, Leeann; Herbert, James D.; Forman, Evan M.; Moitra, Ethan; Farrow, Victoria (2008): The assessment of present-moment awareness and acceptance: the Philadelphia Mindfulness Scale. In Assessment 15 (2), pp. 204-223. DOI: 10.1177/1073191107311467.

DJIKIC, Maja (2014): Art of Mindfulness: Integrating Eastern and Western Approaches Art of Mindfulness: Integrating Eastern and Western Approaches. In Amanda le, Christelle T. Ngnoumen, 
Ellen J. Langer (Eds.): The Wiley Blackwell Handbook of Mindfulness. With assistance of Kristina Niedderer. Chichester, UK: John Wiley \& Sons, Ltd, pp. 139-148. Available online at https://www.researchgate.net/publication/262336620_Art_of_Mindfulness_Integrating_Eastern_ and_Western_Approaches, checked on 2017.

FELDMAN, Greg; Hayes, Adele; Kumar, Sameet; Greeson, Jeff; Laurenceau, Jean-Philippe (2007): Mindfulness and Emotion Regulation: The Development and Initial Validation of the Cognitive and Affective Mindfulness Scale-Revised (CAMS-R). In J Psychopathol Behav Assess 29 (3), pp. 177-190. DOI: 10.1007/s10862-006-9035-8.

GALOR, Sharon (2012): Clarifying states and traits. Available online at https://drsharongalor.wordpress.com/2012/07/09/clarifying-states-and-traits/, checked on 11/12/2017.

GUNARATANA, Henepola (2012): The Four Foundations of Mindfulness in Plain English: Wisdom Publications.

IE, Amanda; Ngnoumen, Christelle T.; Langer, Ellen J. (Eds.) (2014): The Wiley Blackwell Handbook of Mindfulness. With assistance of Kristina Niedderer. Chichester, UK: John Wiley \& Sons, Ltd.

KRASNER, Michael S.; Epstein, Ronald M.; Beckman, Howard; Suchman, Anthony L.; Chapman, Benjamin; Mooney, Christopher J.; Quill, Timothy E. (2009): Association of an educational program in mindful communication with burnout, empathy, and attitudes among primary care physicians. In JAMA 302 (12), pp. 1284-1293. DOI: 10.1001/jama.2009.1384.

KABAT-ZINN, J. (1982). An outpatient program in behavioral medicine for chronic pain patients based on the practice of mindfulness meditation: theoretical considerations and preliminary results. Gen. Hosp. Psychiatry 4, 33-47

KABAT-ZINN, J. (1990). Full Catastrophe Living: Using the Wisdom of Your Body and Mind to Face Stress, Pain and Illness. New York, NY: Delacorte.

KABAT-ZINN, J. (1994). Wherever you go, there you are: Mindfulness meditation in everyday life. New York: Hyperion.

KABAT-ZINN, Jon (2003): Mindfulness-Based Interventions in Context. Past, Present, and Future. In Clinical Psychology: Science and Practice 10 (2), pp. 144-156. DOI: 10.1093/clipsy.bpg016.

LANGER, Ellen J. (1989) Mindfulness. Addison-Wesley, Reading, MA (1989)

LANGER, Ellen J. (1992): Matters of mind. Mindfulness/mindlessness in perspective. In Consciousness and Cognition 1 (3), pp. 289-305. DOI: 10.1016/1053-8100(92)90066-J.

LANGER, Ellen J. (2000): Mindful Learning. In Current directions in psychological science 9 (6), pp. 220-223, checked on 9/19/2017.

LANGER, Ellen J.; Moldoveanu, Mihnea (2000): The Construct of Mindfulness. In Journal of Social Issues 56. DOI: 10.1111/0022-4537.00148.

LAU, Mark A.; Bishop, Scott R.; Segal, Zindel V.; Buis, Tom; Anderson, Nicole D.; Carlson, Linda et al. (2006): The Toronto Mindfulness Scale: development and validation. In Journal of clinical psychology 62 (12), pp. 1445-1467. DOI: 10.1002/jclp.20326.

LIM, Daniel; Condon, Paul; DeSteno, David (2015): Mindfulness and compassion. An examination of 
mechanism and scalability. In PloS one 10 (2), pp. e0118221. DOI: 10.1371/journal.pone.0118221.

MEDVEDEV, Oleg N.; Krägeloh, Christian U.; Narayanan, Ajit; Siegert, Richard J. (2017): Measuring Mindfulness: Applying Generalizability Theory to Distinguish between State and Trait. In Mindfulness 8 (4), pp. 1036-1046. DOI: 10.1007/s12671-017-0679-0.

NHAT HANH, T. (1987): Miracle of Mindfulness. Manual on Meditation. N.e: Beacon P., U.S.

Niedderer, Kristina (2004): Designing the Performative Object: a study in designing mindful interaction through artefacts. Doctor of Philosophy. University of Plymouth, Plymouth.

NIEDDERER, Kristina (2014): Mediating Mindful Social Interactions through Design. In Amanda le, Christelle T. Ngnoumen, Ellen J. Langer (Eds.): The Wiley Blackwell Handbook of Mindfulness. With assistance of Kristina Niedderer. Chichester, UK: John Wiley \& Sons, Ltd. Available online at http://onlinelibrary.wiley.com/book/10.1002/9781118294895, checked on 2017.

NIEDDERER, Kristina; Isabelle Tournier; Dons Colesten-Shields; Michael Craven; Julie Gosling; Ben Salter et al. (2017): Designing with and for People with Dementia: Developing a Mindful Interdisciplinary Co-Design Methodology. In : Proceeding of 7th International Congress of the International Association of Societies of Design Research (IASDR).

OSTAFIN, Brian D.; Robinson, Michael D.; Meier, Brian P. (2015): Handbook of Mindfulness and SelfRegulation. With assistance of Patricia P. Schultz and Richard M. Ryan. 1st ed. 2015. New York, NY: Springer New York.

PIRSON, Michael; Langer, Ellen J.; Bodner, Todd; Zilcha, Sigal (2012): The Development and Validation of the Langer Mindfulness Scale - Enabling a Socio-Cognitive Perspective of Mindfulness in Organizational Contexts. In SSRN Journal. DOI: 10.2139/ssrn.2158921.

ROJAS; English; Young; Spencer (2016): Bridging Mindfulness and Design. In Pereira, Fonseca, Martins, Arezes and Félix (Ed.): Proceedings UD16 - 5th PhD in Design Research Meeting. 5th PhD in Design Research Meeting. University of Aveiro - Portugal, 22 - 23.07.2016. Pereira, Fonseca, Martins, Arezes and Félix. University of Aveiro - Portugal: UA Editora.

ROJAS; F, English; S, Young; R \& Spencer; N (2015): Making Mindfulness explicit in Design Education. In Robin Vande, Erik Bohemia, Ingvild Digranes (Eds.): Learnxdesign. Proceedings of the 3rd International Conference for Design Education Researchers, 28-30 June 2015, Chicago, II, USA. Aalto: University [etc.].

ROJAS; F, English; S, Young; R \& Spencer; N (Eds.) (2017): A design-relevant mindfulness device. 12th EAD Conference, 12-14 April 2017. rome.

SHAPIRO, Shauna L.; Schwartz, Gary E.; Bonner, Ginny (1998): Effects of Mindfulness based stress reduction on medical premedical students. In Journal of Behavioral Medicine 21 (6), pp. 581-599. DOI: 10.1023/A:1018700829825.

SHAPIRO, Shauna L.; Carlson, Linda E.; Astin, John A.; Freedman, Benedict (2006): Mechanisms of mindfulness. In Journal of clinical psychology 62 (3), pp. 373-386. DOI: 10.1002/jclp.20237.

Sliwinski, Jacek; Katsikitis, Mary; Jones, Christian Martyn (2015): Mindful Gaming: How Digital Games Can Improve Mindfulness. In Julio Abascal, Simone Barbosa, Mirko Fetter, Tom Gross, Philippe Palanque, Marco Winckler (Eds.): Human-Computer Interaction - INTERACT 2015, vol. 9298. Cham: Springer International Publishing (Lecture Notes in Computer Science), pp. 167-184. 
SPIELBERGER, C. D., \& Sydeman, S. J. (1994). State-trait anxiety inventory and state-trait anger expression inventory. In M. E. Maruish (Ed.), The use of psychological testing for treatment planning and outcome assessment (pp. 292-321). Lawrence Erlbaum Associates

TANAY, Galia; Bernstein, Amit (2013): State Mindfulness Scale (SMS): development and initial validation. In Psychological assessment 25 (4), pp. 1286-1299. DOI: 10.1037/a0034044.

VAGO, David R.; Silbersweig, David A. (2012): Self-awareness, self-regulation, and selftranscendence (S-ART): a framework for understanding the neurobiological mechanisms of mindfulness. In Frontiers in human neuroscience 6, p. 296. DOI: 10.3389/fnhum.2012.00296.

VANDE, Robin; Bohemia, Erik; Digranes, Ingvild (Eds.) (2015): Learnxdesign. Proceedings of the 3rd International Conference for Design Education Researchers, 28-30 June 2015, Chicago, II, USA. Aalto: University [etc.].

WALACH et at (2006): Measuring mindfulness-the Freiburg Mindfulness Inventory (FMI). In Personality and Individual Differences 40 (8), pp. 1543-1555. DOI: 10.1016/j.paid.2005.11.025.

YEGANEH, B.; Kolb, D. (2009): Mindfulness and Experiential Learning. In OD PRACTITIONER 41 (3), pp. 8-14. Available online at http://learningfromexperience.com/media/2010As 